\author{
„DIE NEUE BIOSTOFFVERORDNUNG UND DIE DARAUF \\ AUFBAUENDEN TRBA WERDEN INSGESAMT KLARER \\ STRUKTURIERT UND LESBAR SEIN.
}
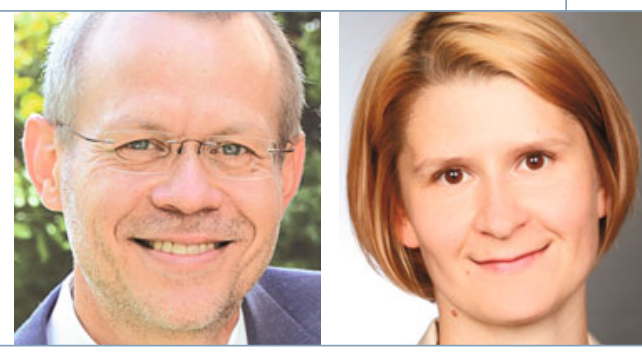

Peter Kämpfer und Silke Hopf

\title{
Die Biostoffverordnung wird novelliert
}

Die Biostoffverordnung ${ }^{1}$ (BioStoffV) als konkretisierende Verordnung zum Arbeitsschutzgesetz befasst sich mit berufsbedingten Tätigkeiten mit biologischen Arbeitsstoffen und enthält Regelungen zum Schutz der Beschäftigten, die mit biologischen Arbeitsstoffen oder Biostoffen umgehen bzw. diesen ausgesetzt sind.

Das Bundesministerium für Arbeit und Soziales hat bei der Neufassung der BioStoffV neue Erkenntnisse zur praktischen Anwendung aufgegriffen und die Verordnung an wissenschaftliche und technische Entwicklungen angepasst. Mit der Neufassung wird die EU-Nadelstichrichtlinie ${ }^{2}$, die Regelungen zur Vermeidung von Verletzungen durch scharfe oder spitze Instrumente im Krankenhaus- und Gesundheitssektor beinhaltet, in nationales Recht umgesetzt.

Das Bundeskabinett hat der Verordnung mit den Maßgaben des Bundesrates am 3. Juli 2013 zugestimmt; am 23.7.2013 trat die neue Biostoffverordnung in Kraft.

Wesentliche Neuerungen der BioStoffV sind:

- Verwendung stichsicherer Instrumente im Gesundheitsdienst, soweit technisch möglich und zur Vermeidung einer Infektionsgefährdung erforderlich.

- Verzicht auf die formalen Anforderungen zur Klassifizierung der Infektionsgefährdung (Schutzstufenzuordnung) bei nicht gezielten Tätigkeiten, bei denen die sensibilisierenden oder toxischen Wirkungen der Biostoffe im Vordergrund stehen. Dies betrifft $\mathrm{u}$ a. Tätigkeiten in der Landwirtschaft, der Abfall- und Abwasserbehandlung, der Kompostierung und dem Sanierungsgewerbe.

- Konkretisierung der Anforderungen an die Fachkunde in Abhängigkeit von der durchzuführenden Aufgabe und Höhe der Gefährdung bei Tätigkeiten mit Biostoffen.

- Ersatz des bisherigen Anzeigeverfahrens für Tätigkeiten mit hochpathogenen Biostoffen durch ein Erlaubnisverfahren.
- Anpassung der Anhänge II und III, die zusätzliche Schutzmaßnahmen für Tätigkeiten in Laboratorien bzw. in der Biotechnologie beinhalten, an den Stand von Wissenschaft und Technik.

Im Rahmen der Neufassung der BioStoffV ist die Überarbeitung einiger Technischer Regeln für Biologische Arbeitsstoffe (TRBA) und die Erarbeitung einer TRBA zu Fachkundeanforderungen nach BioStoffV erforderlich. Unmittelbar sind vier TRBA betroffen:

(1) Die TRBA 100 „Schutzmaßnahmen für Tätigkeiten mit biologischen Arbeitsstoffen in Laboratorien“ wurde bereits im April 2013 vom Ausschuss für Biologische Arbeitsstoffe (ABAS) beschlossen und wird überarbeitet voraussichtlich im Herbst 2013 bekannt gegeben. Diese TRBA wurde mit Bezug auf den Abschnitt „Gefährdungsbeurteilung“ um eine Beispielsammlung für nicht gezielte Tätigkeiten und um einen Abschnitt zu biologischen Arbeitsstoffen mit sensibilisierenden, toxischen oder sonstigen die Gesundheit schädigenden Wirkungen erweitert.

(2) Die Überarbeitung der TRBA 250 „Biologische Arbeitsstoffe im Gesundheitswesen und in der Wohlfahrtspflege" ist weit fortgeschritten und wird voraussichtlich Anfang 2014 bekannt gegeben. Diese TRBA findet Anwendung auf Tätigkeiten mit biologischen Arbeitsstoffen in Arbeitsbereichen des Gesundheitswesens und der Wohlfahrtspflege. Hierbei wurde die Maßnahmenhierarchie klarer herausgestellt, die notwendige Fachkunde des Durchführenden klarer beschrieben sowie Institutionen benannt, die im Besonderen Informationen zu Infektionserregern und Gefährdungsbeurteilung vorhalten. Darüber hinaus enthält die TRBA klarere Definitionen der Schutzstufen 1 bis 4 und prägnantere Beispiele.

(3) Die Erarbeitung einer TRBA zu Fachkundeanforderungen nach BioStoffV ist ebenfalls weit fortgeschritten; mit der Bekanntgabe ist im Frühsommer 2014 zu rechnen. Diese TRBA wird detaillierte Angaben zu Fach-

\footnotetext{
1 Verordnung über Sicherheit und Gesundheitsschutz bei Tätigkeiten mit Biologischen Arbeitsstoffen

2 Richtlinie 2010/32/EU, http://eur-lex.europa.eu/LexUriServ/LexUriServ.do?uri=0J:L:2010:134:0066:0072:DE:PDF
}

kundevoraussetzungen und Fachkundeanforderungen enthalten, wobei diese nach derzeitigem Diskussionsstand differenziert nach unterschiedlichen Tätigkeiten bzw. Tätigkeitsbereichen dargestellt werden sollen (z. B. Tätigkeiten ohne Schutzstufenzuordnung, in den Schutzstufen 1 oder 2 bzw. 3 oder 4).

(4) Auch die Bekanntgabe der überarbeiteten TRBA 400 „Handlungsanleitung zur Gefährdungsbeurteilung und für die Unterrichtung der Beschäftigten bei Tätigkeiten mit biologischen Arbeitsstoffen“ wird für die zweite Jahreshälfte 2014 angestrebt.

Die neue Biostoffverordnung und die darauf aufbauenden TRBA werden insgesamt klarer strukturiert und lesbar sein und mit Bezug auf die Tätigkeiten mit biologischen Arbeitsstoffen detailliertere und praxisbezogene Anforderungen enthalten.

Weitere Informationen zur Neufassung der Biostoffverordnung, dem ABAS und den TRBA finden Sie unter www.baua.de/abas.

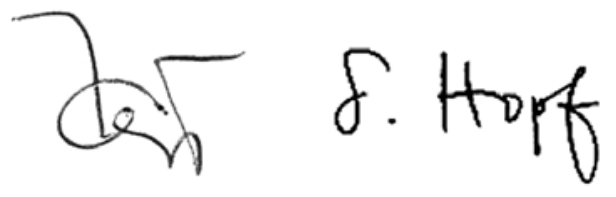

Peter Kämpfer, Vorsitzender des ABAS, und Silke Hopf, Geschäftsführung des ABAS

Korrespondenzadressen:

Prof. Dr. Dr.-Ing. Peter Kämpfer

Justus-Liebig-Universität Gießen

Institut für Angewandte Mikrobiologie

Heinrich-Buff-Ring 26

D-35392 Gießen

Tel.: 0641-9937352

Fax: 0641-9937359

peter.kaempfer@umwelt.uni-giessen.de

Silke Hopf

Bundesanstalt für Arbeitsschutz und Arbeitsmedizin

Nöldnerstraße 40-42

D-10317 Berlin

Tel.: 030-51548 4321

abas@baua.bund.de

www.baua.de/abas 